\title{
15 Bedeutung bildgebender Verfahren
}

Die grundlegenden und zentralen trainingswissenschaftlichen Annahmen zur sportlichen Trainierbarkeit von Menschen basieren auf biowissenschaftlichen Erkenntnissen zur phänotypischen (motorischen, metabolischen, morphologischen, synaptischen und neuronalen) Plastizität. Ohne die Existenz der Plastizität organischer Strukturen und Funktionen wären sportliches Training, Bewegungs- und Körperbildung weitestgehend gegenstands- und sinnlos. Die biowissenschaftlichen Erkenntnisse zur genotypischen und phänotypischen Plastizität sind in den letzten Jahrzehnten, nicht zuletzt durch die Einbeziehung bildgebender Verfahren und die Durchführung zahlreicher Interventionsstudien angewachsen und gut belegt. Bildgebende Verfahren wie PET, MRT, fMRT, MEG und TMS avancierten zum überzeugenden Beweismittel für die - früher so nicht für möglich gehaltene - neuronale synaptische Plastizität in der Humanontogenese (Kandel 2011; 2015; Baltes et al. 2006). Als evident gilt: Neubildung neuer Kontaktstellen, selektive Eliminierung bestehender Kontaktstellen, Vergrößerung oder Verkleinerung von Kontaktstellen, Verstärkung der synaptischen Übertragungseffektivität, Abschwächung der synaptischen Übertragungsaktivität (Bock 2017). Die materielle Grundlage des Lernens wurde überzeugend identifiziert und die neuronale Erzeugung des Mentalen nachgewiesen. Trotz aller noch bestehender Erklärungslücken bezüglich der inhaltlichen Bestimmtheit des Neuronalen und der Entstehung des subjektiven Erlebnisgehalts mentaler Zustände (sogenannter "Qualia“), sprechen die vorliegenden Befunde dafür, dass immaterielles, „rein“ Mentales nicht existiert.

Auf die Bedeutung der strukturellen Neuroplastizität für Sport und Training weisen Ragert und Carius (2017) hin. Trainingsinduzierte Strukturveränderungen im Gehirn durch das Bewältigen motorischer Balancieraufgaben sind nachgewiesen. 